\title{
Biological Tissue Characterization using Optical Backscattering Technique to detect the Presence of Abnormalities
}

\author{
C. Jim Elliot, S. Anitha, R. Vijayakumar, A. Akhila, R. Pradeepa
}

\begin{abstract}
: we are using imaging modality to observe and acquire the information from the tissue. The backscattered intensity variation depends upon the composition, color and blood flow of the tissue. The optical parameters of the tissue are determined by using serial monitor in Arduino. Thus the tissues are treated of diameter $0.1,0.2$ and $0.3 \mathrm{~cm}$ and placed at different depth. From this method, we can diagnose the abnormalities in the skin tissue/phantom. Photon depth distribution (anisotropy) and surface intensity differs which depends on various tissues. Thus scanned in horizontal position we have to find the depth. By peak intensity analysis, we have to determine the type, location and size of the tissue variation. Backscattering of laser from the tissue is measured by using optic probe. The backscattering parameter gives some information about the tissue because the light beam gets penetrate in some existence.
\end{abstract}

Keywords : Backscattered intensity, Laser reflectance, Optical Phantom, Optical parameters, Tissue Characterization

\section{OBJECTIVE}

Image is defined as the visual perception of an object. Image is a two dimensional representation of an object. It consists of more number of pixels to form an image. Medical imaging is a technique to create a visual representation of parts / organs of the body. They help to diagnose and monitor the health condition. There are many different types of imaging modalities such as X-ray, CT scan, MRI and ultrasound techniques etc. X-ray exposure can cause increased risk of cancer. In CT scan we use higher doses of radiation when compared to X-Ray. The injection of a dye causes renal problem or allergy etc. MRI procedure is lengthy, noisy and if any slight movement occurs the procedure has to be repeated. The existing methods for tissue

Revised Manuscript Received on December 16, 2019.

* Correspondence Author

C. Jim Elliot*, Department of Biomedical Engineering, Kalasalingam Academy of Research \& Education, VirudhuNagar, India. Email: jimelliot.c@gmail.com

S. Anitha, Department of Biomedical Engineering, Karunya Institute of Technology \& Sciences, Coimbatore, India. Email: anithaanu43363@gmail.com

A. Akhila, Department of Biomedical Engineering, Kalasalingam Academy of Research \& Education, VirudhuNagar, India. Email: akhilaakash3@gmail.com

R. Vijayakumar, Department of Biomedical Engineering, Kalasalingam Academy of Research \& Education, VirudhuNagar, India. Email: vijayakumar20036@gmail.com

R. Pradeepa, Department of Biomedical Engineering, Kalasalingam Academy of Research \& Education, VirudhuNagar, India. Email: rpradeepa@gmail.com characterization such as histology, microbiology and biopsy have their methodological limitations. Histopathology is an invasive technique which helps to study the structure and function of cells, tissues etc. Biopsy is also an invasive technique in which the sample of tissue is taken from the body in order to examine the abnormal tissues or tumors using microscopy. It is a painful process. The earlier methods of tissue characterization have several disadvantages such as time consumption, low accuracy, invasiveness etc. To overcome this we use laser methodology for imaging technique. It is non-ionizing radiation method. This methodology provides solution to the problem using currently available laser diagnosis for tissue characterization. It provides valuable information about abnormal tissues present in our body.

The objective of our work is to determine the optical parameters and used it for reconstruction of surface reflectance and photon depth distribution image. The abnormalities of tissue are determined by varying sizes inside the normal tissue. The optical properties are transmission, reflection, scattering, absorption and anisotropy.

Transmission states that when the light rays hit the medium and it gets transmitted into other medium. The light rays strike the medium it gets reflected from the medium that is known as reflection. During collision, the direction of particle motion gets changed, that is called scattering. Scattering means the beam of light which hit the subject and it gets scattered in all direction. Anisotropy means dissimilar, when we measure the material at different axes of physical or mechanical properties (conductivity, refractive index, absorbance, etc...). It is directionally dependent. Example: The light passing through a polarizer.

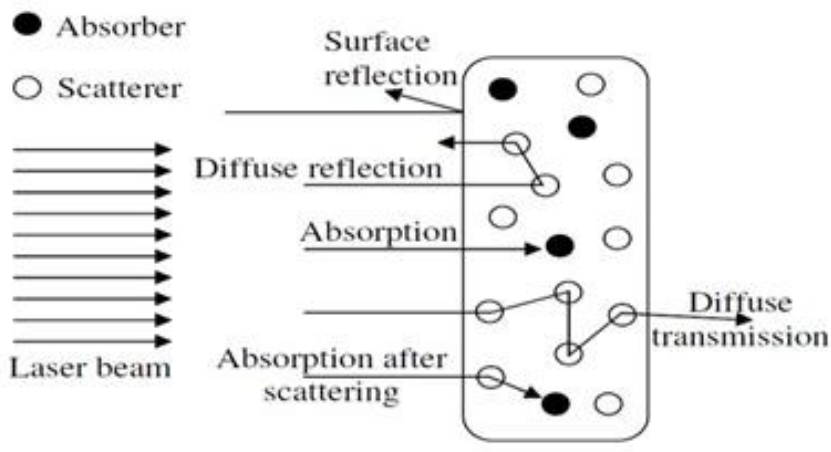

Fig. 1. Optical Properties

Published By: 
The optical parameters employed in our work are absorption coefficient, scattering coefficient, refractive index and anisotropy factor. Absorption coefficient measure of the rate of decrease in intensity as light passes through a given substances, the fraction of incident energy absorbed per unit area. Refractive index describes that in a medium, how fast the light generation process will happen. It is denoted as „, $\mathrm{n}^{\mathrm{ee}}$. The formula for refractive index is $n=c / v$, whereas $c$ is the speed of light and $v$ is the velocity of light

Skin is the outer covering of a human body. It is a type of soft tissue. It is one of the sensory organs which present in our body. Skin consists of three layers (Epidermis, Dermis and Subcutaneous layer). Absorption rate in skin depends on amount of chromophores. Epidermis consists of melanin pigment, which acts as natural absorbing chromophores. If the presence of melanin is higher, the absorption rate also higher. Dermis composed of collagen fiber, which holds large amount of water. It maintains the turbidity of our skin. It consists of blood vessels and nerves. Collagen fibers act as a scattering element in skin. Absorption rate of both oxy and deoxy-haemoglobin decreases gradually for longer wavelength (above $577 \mathrm{~nm}$ ). Subcutaneous layer is the inner layer of the skin. It is otherwise known as hypodermis. It is mostly comprised of adipose tissue called fat tissues. Adipose tissue acts an absorber. If absorption of light increases the scattering decreases and vice versa. Application of laser in characterization of tissue is an emerging field of diagnostic medicine. In this growing healthcare sector, laser based techniques have been brought into existence and applied in different field of medicines such as oncology, ophthalmology, dentistry, dermatology, etc. The variations of the tissue characterization are differing from person to person, site to site or even time to time on one site.

\section{MATERIALS \& METHODS}

\section{A. Light Emitting Diode}

It is a semiconductor material. It consists of anode and cathode. Anode acts as positive terminal and Cathode acts as negative terminal. It emits light when an electric current is applied in the forward direction. It works under the principle of electroluminescence. The color of the light is determined by the energy required for electrons to cross the band gap of the semiconductor. The output power of led is $5 \mathrm{~mW}$ and the wavelength ranges from 360 to $950 \mathrm{~nm}$. The red led consists of $635 \mathrm{~nm}$ wavelength. It operates between 1.8 to 5 voltage ranges.

\section{B. Transimpedance Amplifier (OPT101)}

OPT101 is an integrated circuit in which the combination of both the transimpedance amplifier and photodiode on a single chip. It eliminates some problems

\begin{tabular}{|c|c|c|c|c|}
\hline Tissue & Layers & $\begin{array}{c}\text { Absorption } \\
\text { coefficient } \\
\left(\mathbf{m m}^{-1}\right)\end{array}$ & $\begin{array}{c}\text { Scattering } \\
\text { coefficient } \\
\left(\mathbf{m m}^{-1}\right)\end{array}$ & Anisotropy \\
\hline $\begin{array}{c}\text { Skin } \\
\text { tissue }\end{array}$ & Epidermis & 9.15 & 55.48 & 0.787 \\
\hline $\begin{array}{c}\text { Breast } \\
\text { Tissue }\end{array}$ & $\begin{array}{c}\text { Adipose } \\
\text { tissue }\end{array}$ & 0.99 & 294.1 & 0.972 \\
\hline
\end{tabular}

Table 1. Optical Parameters

such as noise pick-up, gain peaking and leakage error in a result of capacitance stray. With light intensity increasing output voltage linearly. The single or dual power supply operation is performed by the amplifier in the integrated component. Photodiode is used to convert the light into current, which is type of semiconductor device. Transimpedance amplifier is a type of operational amplifier, which converts the electrical current from the photodiode, is converted into an electric voltage

\section{METHODOLOGY}

The methodology consists of a LED of power $5 \mathrm{~mW}$ which has an operating range of $655 \mathrm{~nm}$. The photodiode is connected with Arduino for data acquisition which is interfaced with PC. LED acts as a source and the photodiode acts as a detector. From the image we can determine the absorption coefficient, scattering coefficient and anisotropy parameters. It is a non-invasive technique and non-ionizing method.

\section{A. Phantom Preparation}

Take a wax pellets and melts them at $70-80^{\circ} \mathrm{C}$. Keep the melted wax in rectangular box. Place the measured content in the layer of wax at different depth. The surface reflectance measured after solidification by removing from the cast cylinder. It is an tissue mimicking phantom.

\section{B. Working Principle}

LED acts as a source of $655 \mathrm{~nm} 5 \mathrm{~mW}$. It has two pin. The pin1 is acting as an input voltage $\mathrm{V}_{\mathrm{cc}}$ of $1.8 \sim 5 \mathrm{~V}$, which is connected with variable resistor of $10 \mathrm{k} \Omega$. The pin 2 is grounded .It gives stable output power. OPT101 is an integrated circuit in which the combination of both the transimpedance amplifier and photodiode on a single chip. The input voltage is $2.7 \sim 36 \mathrm{~V}$. It eliminates noise pick-up, gain peaking and leakage error in a result of capacitance stray. Photodiode is used to convert the light into current.

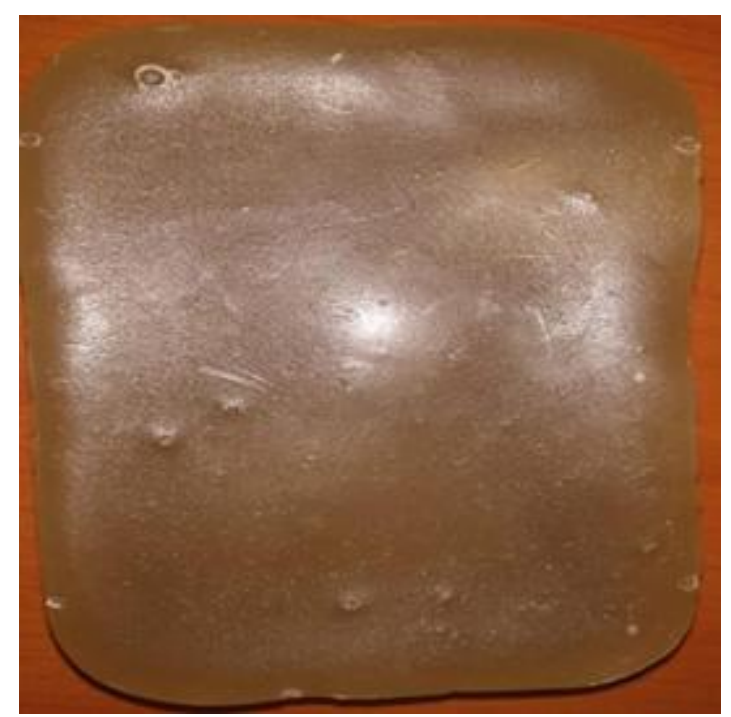

Fig. 2. Optical Tissue Equivalent

Published By: 
Transimpedance amplifier which converts the electrical current from

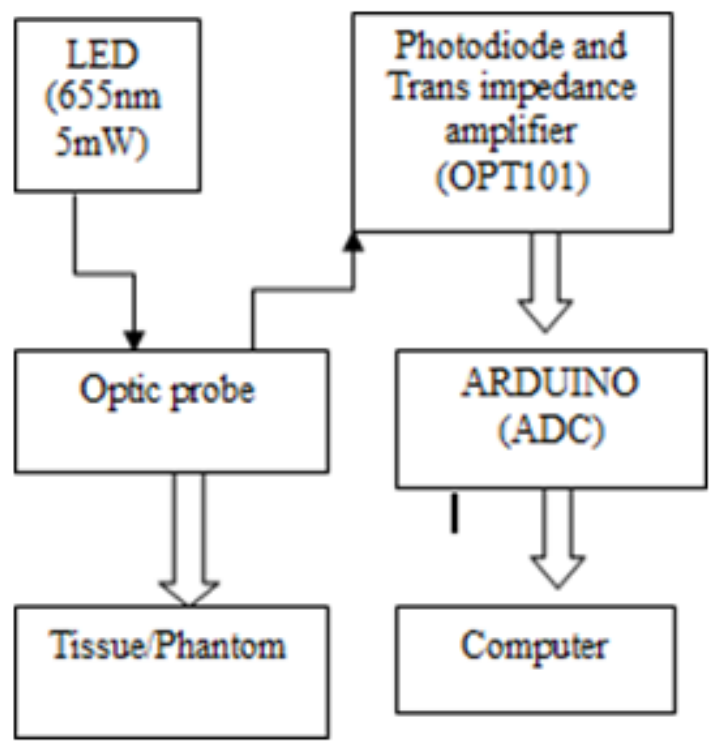

Fig. 3. Block Diagram

the photodiode is converted into an electrical voltage. With light intensity increasing output voltage linearly. The single or dual power supply operation is performed by the amplifier in the integrated component. Thus we placed the setup inside the darkroom to remove the noise. The output voltage from the detector is varied which depends on the tissue. Thus we use one source and three detectors to obtain the characterization value. The input power from the Arduino of $3.3 \mathrm{~V}$ is given to the source and the detector. The output from the detector is given to the Arduino as an analog input. The output from the detector is obtained in the serial monitor and serial plotter.

\section{RESULTS \& DISCUSSION}

This paper shows the backscattering from the tissue volume. After the beam entry in the tissue, it gets broadened because of multiple interactions. These characterizations do not only depend on photon distribution but also the location, absorption, scattering and refractive index of the tissue.

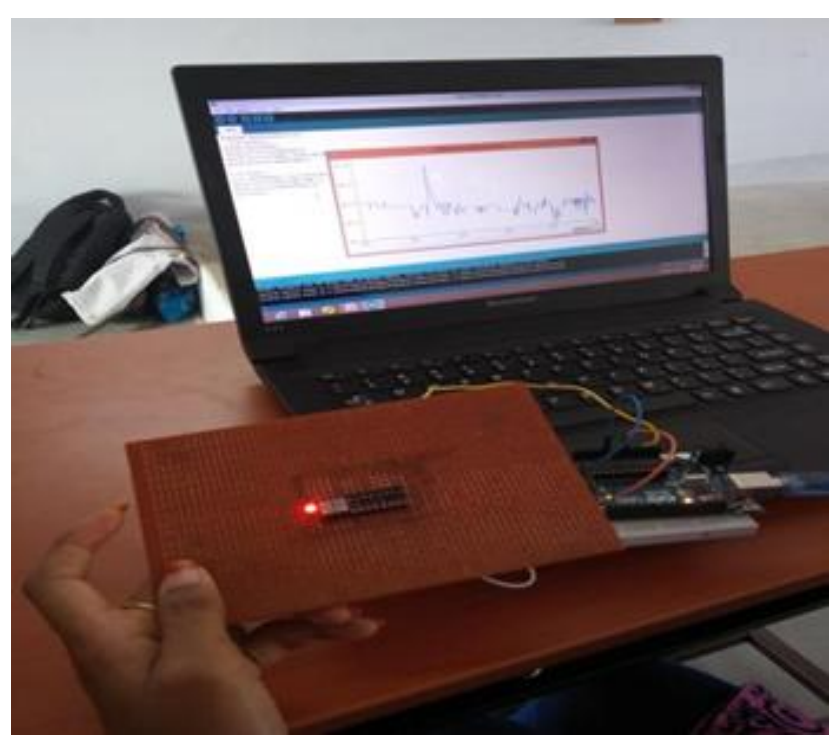
depends upon type, size, color, composition and blood

Fig. 3. Working Setup

\begin{tabular}{|c|c|}
\hline Sl. No. & Output values \\
\hline 1 & 138 \\
\hline 2 & 114 \\
\hline 3 & 120 \\
\hline 4 & 126 \\
\hline 5 & 131 \\
\hline 6 & 136 \\
\hline 7 & 141 \\
\hline 8 & 146 \\
\hline 9 & 150 \\
\hline 10 & 154 \\
\hline 11 & 157 \\
\hline 12 & 160 \\
\hline 13 & 162 \\
\hline 14 & 163 \\
\hline 15 & 164 \\
\hline 16 & 164 \\
\hline 17 & 164 \\
\hline 18 & 163 \\
\hline 19 & 161 \\
\hline
\end{tabular}

Table 2. Output Values

The maximum depth of penetration in tissue is defined as the range falls of intensity from unity to 0.0001 . This parameter depends on the conditions, sample preparations and techniques. The formation of photon distribution depends on anisotropic parameter.

\section{CONCLUSION}

In anisotropic condition, the photon backscattering gives better information with different layers of tissues. This method helps us to verify the valuable information of diagnosis and treatment in biomedical applications.

\section{REFERENCES}

1. D.Kumar \& Megha Singh, "Characterization and Imaging of Compositional Variation in Tissues",IEEE Transactions on Biomedical Engineering, vol. 50, No. 8, August 2003,pp.1012-1019.

2. P.S.Pandian \& Megha Singh, "Localization and characterization of tissue changes by laser backscattering imaging and Monte Carlo simulation", Indian journal of experimental Biology, vol. 50, No. 8, August 2003.

3. R.Srinivasan \&Meghan Singh, "Laser Backscattering and Transillumination Imaging of Human Tissues and their Equivalent Phantoms", IEEE Transactions, vol. 42, No. 1, 2008,pp.724-730.

4. Fuks, David, Angelo Pierangelo, Pierre Validire, Marine Lefevre, Abdelali Benali, Guillaume Trebuchet, Aline Criton, and Brice Gayet. "Intraoperative confocal laser endomicroscopy for real-time in vivo tissue characterization during surgical procedures." Surgical endoscopy 33, no. 5 (2019),pp.1544-1552.

5. Martin, Chris, and Adela Ben-Yakar. "Determination of scattering properties and damage thresholds in tissue using ultrafast laser ablation." Journal of biomedical optics 21, no. 11 (2016),pp.115004.

6. Wally, Zena J., et al. "Selective laser melting processed Ti6Al4V lattices with graded porosities for dental applications." Journal of the mechanical behavior of biomedical materials 90 (2019),pp. 20-29.

Published By:

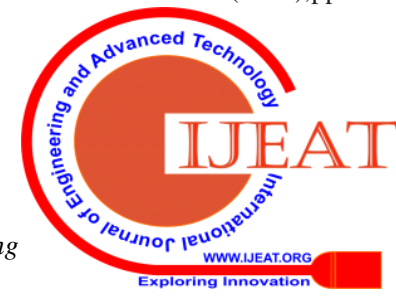


7. Bashkatov, Alexey N., Elina A. Genina, and Valery V. Tuchin. "Optical properties of skin, subcutaneous, and muscle tissues: a review." Journal of Innovative Optical Health Sciences 4, no. 01 (2011),pp.9-38.

8. Tuchin, Valery V. "Tissue optics and photonics: biological tissue structures." Journal of Biomedical Photonics \& Engineering 1.1 (2015).

9. Chacko, S., and M. Singh. "Multi-layer imaging of human organs by measurement of laser backscattered radiation." Medical \& biological engineering \& computing 37, no. 3 (1999),pp- 278.

10. Cheong, Wai-Fung, Scott A. Prahl, and Ashley J. Welch. "A review of the optical properties of biological tissues." IEEE journal of quantum electronics 26, no. 12 (1990): 2166-2185.

\section{AUTHORS PROFILE}

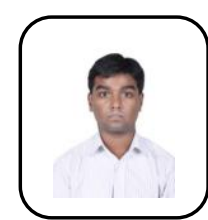

Mr. C. Jim Elliot is working as Assistant Professor in the department of Biomedical Engineering at Kalasalingam Academy of Research \& Education and pursuing his $\mathrm{PhD}$ research in optical imaging. He has worked as Senior Research Fellow in a DST funded project.

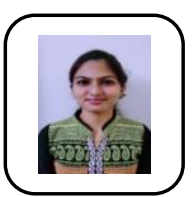

Ms. S. Anitha is a PG scholar from Biomedical Instrumentation at Karunya Institute of Technology \& Sciences. She received her B. Tech. in Biomedical Engineering from Kalasalingam Academy of Research \& Education during the year 2019. She has published two research articles in reputed international journals and conferences. Her current research interest includes signal, image processing and cancer imaging. She has attended 20 hours online course organized by NPTEL and got certification for Introduction to Mechanobiology. She is aiming to pursue her $\mathrm{PhD}$ in the same domain.

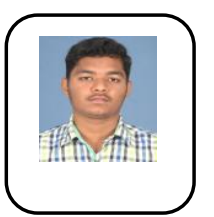

Mr. R. Vijayakumar had completed her B. Tech in Biomedical Engineering at Kalasalingam Academy of Research \& Education during the year 2019.

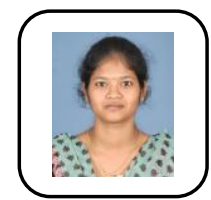

Ms. A. Akhila had completed her B. Tech in Biomedical Engineering at Kalasalingam Academy of Research \& Education during the year 2019.

Ms. R. Pradeepa had completed her B. Tech in Biomedical Engineering at Kalasalingam Academy of Research \& Education during the year 2019. 